\title{
CD99 Gene
}

National Cancer Institute

\section{Source}

National Cancer Institute. CD99 Gene. NCI Thesaurus. Code C102939.

This gene plays a role in the cell adhesion process. 